\title{
Prevalence of and risk factors for cerebral microbleeds in a general Japanese elderly community
}

Tomohiro Yubi, MD, Jun Hata, MD, PhD, Tomoyuki Ohara, MD, PhD, Naoko Mukai, MD, PhD, Yoichiro Hirakawa, MD, PhD, Daigo Yoshida, PhD, Seiji Gotoh, MD, PhD, Naoki Hirabayashi, MD, PhD, Yoshihiko Furuta, MD, Tetsuro Ago, MD, PhD, Takanari Kitazono, MD, PhD, Yutaka Kiyohara, MD, PhD, and Toshiharu Ninomiya, MD, PhD
Correspondence

Dr. Hata

junhata@eph.med.kyushu-u.ac.jp

Neurology: Clinical Practice June 2018 vol. 8 no. 3 223-231 doi:10.1212/CPJ.0000000000000464

\section{Abstract}

\section{Background}

We investigated the prevalence of and risk factors for cerebral microbleeds (CMBs) in a cross-sectional study of a general population of Japanese elderly.

\section{Methods}

In 2012, brain MRI scanning at $1.5 \mathrm{~T}$ and comprehensive health examination were conducted for 1281 residents aged 65 years or older. CMBs were defined as ovoid hypointensity lesions less than $10 \mathrm{~mm}$ in diameter on $\mathrm{T}^{*}$-weighted images and classified into deep/infratentorial or lobar CMBs. Age- and sex-specific and overall prevalence of CMBs were estimated, and the associations of traditional cardiovascular risk factors and $A P O E$ polymorphism with the presence of CMBs were examined using a logistic regression analysis.

\section{Results}

The crude prevalences of total, deep/infratentorial, and lobar CMBs were $18.7 \%(\mathrm{n}=240), 13.5 \%(\mathrm{n}=173)$, and 9.6\% $(\mathrm{n}=123)$, respectively. The prevalence of total $\mathrm{CMBs}$ was $23.0 \%$ in men and $15.5 \%$ in women and increased with aging in both sexes (both $p$ for trend $<0.01$ ). Hypertension was significantly associated with the presence of both deep/infratentorial and lobar CMBs. Lower serum total cholesterol was a significant risk factor for deep/infratentorial CMBs, but not for lobar CMBs, while $A P O E \& 4$ carriers had a significantly higher likelihood only of lobar CMBs compared with noncarriers.

\section{Conclusions}

Our study suggests that approximately 1 of 5 Japanese elderly people have CMBs, and that risk factors for deep/infratentorial and lobar CMBs are different, indicating the distinct pathologic backgrounds of these lesions.

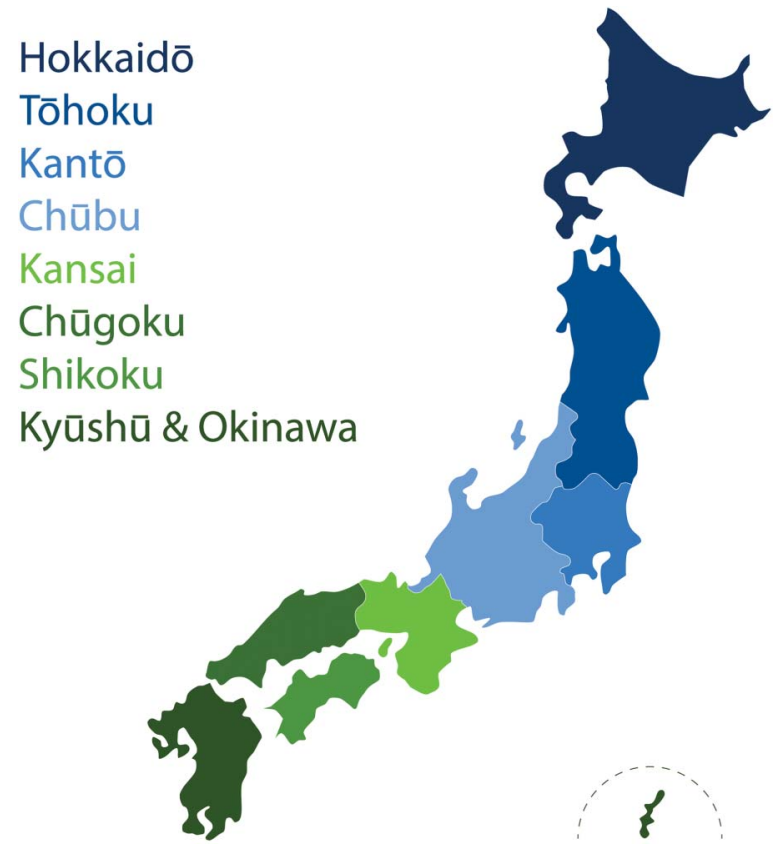


Cerebral microbleeds (CMBs) are round lesions with hypointensity on $\mathrm{T}^{*}$-weighted images of brain MRI. ${ }^{1} \mathrm{CMBs}$ are usually asymptomatic and thus are often identified incidentally in the brain MRI examinations for patients with stroke and dementia. ${ }^{2}$ Histologically, CMBs represent tiny focal collections of hemosiderin adjacent to abnormal small vessels affected by mainly hypertensive or amyloid angiopathy. ${ }^{2} \mathrm{CMBs}$ with hypertensive angiopathy are thought to be mostly localized in deep or infratentorial brain areas, while CMBs with amyloid angiopathy are mainly located in cerebral lobar regions. ${ }^{2}$ Therefore, risk factors for CMBs are likely to differ by their locations. Meanwhile, cerebrovascular disease in Japanese and other Asian populations is known to have a different genetic and environmental background compared with cerebrovascular disease in Western populations. ${ }^{3,4}$ This suggests the possibility of distinct characteristics of $\mathrm{CMBs}$ between populations. Recently, the presence of CMBs has been considered to be an early imaging marker of bleeding-prone angiopathy, and thereby as a predictor of the development of symptomatic stroke, especially intracerebral hemorrhage, and dementia in the elderly. ${ }^{2}$ Therefore, the epidemiologic evidence for CMBs in elderly populations is considered to be useful for understanding and prevention of subsequent stroke and dementia. A number of observational studies performed in various locations around the world have reported the prevalence of $\mathrm{CMBs}^{5-17}$ and their risk factors ${ }^{5-16}$ in adult populations, but there have been limited studies addressing these issues in Western elderly populations, ${ }^{9-12}$ and only a few prevalence studies of Asian elderly populations, including Japanese. The aims of the present study were to estimate the prevalence of $\mathrm{CMBs}$ and to explore their risk factors with consideration of the brain localization of CMBs in a general Japanese elderly population.

\section{Methods}

\section{Study participants}

The Hisayama Study is a prospective cohort study of cerebrocardiovascular diseases established in 1961 in Hisayama, a suburban community of the Fukuoka metropolitan area on Kyushu Island, Japan. The population of the town in 2010 was approximately 8,400 . The age and occupational distributions and nutrient intake levels in the residents of Hisayama have been similar to those in Japan as a whole. ${ }^{18}$ The design of the MRI scan study in the town was previously described in detail. ${ }^{19}$ Briefly, in 2012, a total of 1,906 individuals (93.6\%) among 2,036 residents aged $\geq 65$ years participated in a screening survey for cognitive impairment and activities of daily living (figure e-1, links.lww.com/CPJ/A24). This survey was mainly conducted at the municipal center for health promotion (Hisayama Health C\&C Center) with a rental mobile MRI scanner, but surveys were also performed at home, hospitals, or nursing homes for 539 individuals who could not visit the center due to physical disability, dementia, severe diseases, or other causes. Among the 1,367 participants who visited the center, an MRI examination was performed for 1,342 individuals. In addition, we performed a comprehensive health examination for cardiovascular risk factors in the same year. After excluding 1 individual who refused to participate in the study, 41 who did not participate in the comprehensive health examination, 12 without $\mathrm{T} 2{ }^{*}$-weighted imaging, and 7 without blood sampling, the remaining 1,281 individuals were enrolled in the present study.

\section{Standard protocol approvals, registrations, and patient consents}

This study was conducted with the approval of the Kyushu University Institutional Review Board for Clinical Research. Written informed consent was obtained from all participants.

\section{Brain MRI examination}

In the MRI examination, $\mathrm{T}^{*}$-weighted imaging, T1-weighted imaging, T2-weighted imaging, fluid-attenuated inversion recovery (FLAIR) imaging, and magnetic resonance angiography of the brain were performed using a 1.5T MRI scanner (Intera Pulsar; Philips Medical Systems, Best, the Netherlands) with a multichannel head coil. ${ }^{19} \mathrm{~T}^{*}$-weighted $2 \mathrm{D}$ gradient echo images were acquired with the following measures: repetition time $670 \mathrm{~ms}$, echo time $23 \mathrm{~ms}$, flip angle $18^{\circ}$, field of view $240 \times$ $191 \mathrm{~mm}$, acquisition matrix $256 \times 163$, slice thickness $5 \mathrm{~mm}$, and slice gap $1.5 \mathrm{~mm}$. CMBs were defined as ovoid hypointense lesions on $\mathrm{T} 2{ }^{*}$-weighted images measuring $<10 \mathrm{~mm}$ in diameter and surrounded by brain parenchyma over at least half the circumference of the lesion. Hemorrhagic infarction and physiologic calcification in the globus pallidus, which also showed hypointensity on the $\mathrm{T} 2{ }^{*}$-weighted images, were excluded. Sulcal flow voids from vessels were also ignored. Individuals with CMBs were categorized into a deep/infratentorial CMBs group (located in the basal ganglia, thalamus, brainstem, or cerebellum) and a lobar CMBs group (located in the cortical gray matter or subcortical or periventricular white matter). Individuals with both deep/infratentorial and lobar CMBs were included in both groups. For the sensitivity analysis, the strictly lobar CMBs group included the individuals with lobar CMBs without deep/infratentorial CMBs. The presence and the location of CMBs were determined by 2 stroke neurologists who were blinded to the clinical information (agreement ratio 83.9\%; interrater reliability $\kappa=0.54$ ). If there was a conflicting interpretation, a third investigator read the scan and made a final decision.

Lacunar infarction on MRI was defined as a small ischemic lesion ( $\geq 3$ and $<15 \mathrm{~mm}$ in diameter) in the basal ganglia, thalamus, or brainstem visible on both the T1-weighted image (as a hypointense lesion) and the T2-weighted image (as a hyperintense lesion) with a surrounding hyperintense rim on the FLAIR image, irrespective of the presence or absence of clinical symptoms. The amounts of white matter hyperintensity $(\mathrm{WMH})$ in periventricular and deep white matter regions of the brain were quantified into 4 grades, from grade 0 (absent) to grade 3 (severe), according to the Fazekas scale, ${ }^{20}$ using the FLAIR or T2-weighted images. Positive WMH was defined as grade 2 or $3 \mathrm{WMH}$ in either periventricular or deep white matter or both. 


\section{Other variables}

Blood pressure (BP) was measured 3 times in a sitting position, and the mean values were used in the analysis. Hypertension was defined as a mean $\mathrm{BP} \geq 140 / 90 \mathrm{~mm} \mathrm{Hg}$ or use of antihypertensive agents. Plasma glucose levels were determined by the hexokinase method. Diabetes mellitus was defined as fasting plasma glucose $\geq 7.0 \mathrm{mmol} / \mathrm{L}$ 2-hour postload or postprandial plasma glucose $\geq 11.1 \mathrm{mmol} / \mathrm{L}$ or use of antidiabetic medications. Serum total cholesterol levels were determined enzymatically. Hypercholesterolemia was defined as a serum total cholesterol $\geq 5.69 \mathrm{mmol} / \mathrm{L}$ or use of lipid-lowering agents. Body height and weight were measured in light clothing without shoes, and body mass index (BMI) was defined as the body weight (in kilograms) divided by the height (in meters) squared. Information regarding smoking habits and alcohol intake was selfreported and was categorized as current habitual use or not. Regular exercise was defined as engaging in sports at least 3 times a week during leisure time. Antithrombotic medication included both antiplatelets (aspirin, clopidogrel, ticlopidine, and cilostazol) and anticoagulants (warfarin and direct oral anticoagulants). APOE polymorphism was determined by genotyping 2 single nucleotide polymorphisms (rs429358 and rs7412) using a multiplex PCR-based Invader assay (Third Wave Technologies, Madison, WI) for 1,106 participants with genomic DNA samples. ${ }^{21,22}$ Those who carried at least 1 copy of the $\varepsilon 4$ allele were categorized as APOE $\varepsilon 4$ carriers. The participants with at least 1 copy of the $\varepsilon 2$ allele were defined as $A P O E \varepsilon 2$ carriers. History of symptomatic stroke was defined as any preexisting stroke events. All symptomatic stroke events were adjudicated based on physical examinations and all available clinical information and classified into ischemic stroke, intracerebral hemorrhage, and subarachnoid hemorrhage as described previously. ${ }^{18}$ Dementia was ascertained using the criteria of the DSM-III-R ${ }^{23}$ as described previously. ${ }^{19,24}$

\section{Statistical analysis}

The 95\% confidence intervals (CIs) of the prevalence of $\mathrm{CMBs}$ were estimated using a binominal distribution. The association between age and the prevalence of CMBs was estimated using a logistic regression model, and linearity was assessed by comparing the categorical model with the linear trend model. The interaction between age and sex in the prevalence of CMBs was evaluated by adding an interaction term in the logistic model. The prevalences of CMBs in APOE $\varepsilon 4$ carriers and noncarriers were compared using a $\chi^{2}$ test. The age- and sex-adjusted and multivariable-adjusted odds ratios (ORs) and their $95 \%$ CIs for the presence of any CMBs, deep/infratentorial CMBs, and lobar CMBs were computed using a logistic regression model. In these analyses, the individuals without any CMBs were used as a reference group. Age, sex, hypertension, diabetes, serum total cholesterol, BMI, current smoking, current drinking, regular exercise, use of antithrombotic agents, and APOE polymorphisms ( $\varepsilon 2$ and $\varepsilon 4)$ were included in the multivariableadjusted model. The age- and sex-adjusted ORs and their 95\% CIs for symptomatic ischemic stroke, symptomatic intracerebral hemorrhage, dementia, lacunar infarction on
MRI, and WMH were estimated using a logistic regression model. The differences in the mean values or frequencies of risk factors between the individuals included in and excluded from the study were tested using a $\chi^{2}$ or Student $t$ test, respectively. All analyses were conducted using SAS 9.4 (SAS Institute, Cary, NC). Two-sided $p<0.05$ was considered statistically significant in all analyses.

\section{Data availability}

The datasets generated and analyzed in the present study are not publicly available because they contain confidential clinical and demographic data of the study participants. However, the further information about the datasets is available with permission of the corresponding author (Jun Hata) and the principal investigator of the Hisayama Study (Toshiharu Ninomiya) on reasonable request for purposes of replicating procedures and results.

\section{Results}

Table 1 shows the baseline characteristics of the study participants. The mean age was 75 years (SD 7) and the proportion of men was $43.4 \%$. The prevalences of hypertension and diabetes were $70.7 \%$ and $23.3 \%$, respectively, and the mean value of serum total cholesterol levels was $5.11 \mathrm{mmol} / \mathrm{L}$ (SD 0.93). Among total participants, $19.4 \%$ took an antithrombotic medication, and $18.9 \%$ were $A P O E \& 4$ carriers. Men were more likely to have diabetes, a smoking habit, a drinking habit, and antithrombotic use than women, whereas women had higher serum total cholesterol than men.

Table 2 presents the prevalence of any CMBs, deep/ infratentorial CMBs, and lobar CMBs. Among the 1,281 participants, 240 (18.7\%) had at least $1 \mathrm{CMB}$ on MRI and 109 (8.5\%) had multiple CMBs. The prevalences of deep/ infratentorial CMBs and lobar CMBs were $13.5 \%(\mathrm{n}=173)$ and $9.6 \%(\mathrm{n}=123)$, respectively. There were 56 individuals (4.4\%) who had both deep/infratentorial and lobar CMBs, and 67 individuals (5.2\%) who had strictly lobar CMBs without deep/infratentorial CMBs. The prevalence of any CMBs was $23.0 \%$ in men and $15.5 \%$ in women. The prevalence of any CMBs increased linearly with aging, from 11.8\% (age 65-69 years) to $29.2 \%$ (age 85 years or older), and the same was true for both deep/infratentorial and lobar CMBs ( $p$ for trend $<0.01$ for all, $p$ for nonlinearity $>0.5$ for all). No evidence of an interaction between age and sex was found in the prevalence of any, deep/ infratentorial, or lobar CMBs ( $p$ for interaction $>0.3$ for all).

As shown in table 3, older age, male sex, hypertension, use of antithrombotic agents, and $A P O E \& 4$ genotype were significantly associated with the presence of any CMBs in the ageand sex-adjusted analysis. In the multivariable-adjusted analysis, older age and hypertension remained independent significant risk factors for any $\mathrm{CMBs}$, while the associations of sex, antithrombotic treatment, and APOE $\& 4$ genotype with the presence of any $\mathrm{CMBs}$ were no longer significant. 
Tables 4 and 5 demonstrate the associations of each risk factor with deep/infratentorial CMBs and with lobar CMBs, respectively. In the multivariable-adjusted analysis, older age and hypertension were significantly associated with higher risk of the presence of both deep/infratentorial and lobar CMBs. Lower serum total cholesterol was a significant risk factor for deep/infratentorial CMBs, but not for lobar CMBs. In contrast, $A P O E \varepsilon 4$ carriers had a significantly higher risk of the presence of lobar CMBs than noncarriers, but no clear association was observed between APOE genotype and deep/infratentorial CMBs. Men were more likely to have lobar CMBs than women even after adjustment for potential confounding factors. Similar associations were observed for strictly lobar CMBs without deep/infratentorial CMBs (table e-1, links.lww.com/CPJ/A24).

Table 1 Baseline characteristics of the participants, the Hisayama Study, 2012

\begin{tabular}{|c|c|c|c|}
\hline Variables & $\begin{array}{l}\text { Total } \\
(\mathrm{n}=1,281)\end{array}$ & $\begin{array}{l}\text { Men } \\
(n=556)\end{array}$ & $\begin{array}{l}\text { Women } \\
(\mathrm{n}=725)\end{array}$ \\
\hline Age, y & $75(7)$ & $74(6)$ & $75(7)$ \\
\hline Systolic blood pressure, $\mathrm{mm} \mathrm{Hg}$ & $134(19)$ & $135(19)$ & $134(19)$ \\
\hline Diastolic blood pressure, $\mathrm{mm} \mathrm{Hg}$ & $76(11)$ & $78(11)$ & $75(11)$ \\
\hline Hypertension, \% & 70.7 & 71.6 & 70.0 \\
\hline Antihypertensive agent, $\%$ & 55.8 & 57.1 & 54.8 \\
\hline Diabetes mellitus, \% & 23.3 & 30.2 & 17.9 \\
\hline $\begin{array}{l}\text { Serum total } \\
\text { cholesterol, } \mathrm{mmol} / \mathrm{L}\end{array}$ & $\begin{array}{l}5.11 \\
(0.93)\end{array}$ & $\begin{array}{l}4.80 \\
(0.89)\end{array}$ & $\begin{array}{l}5.35 \\
(0.89)\end{array}$ \\
\hline $\mathrm{BMI}, \mathrm{kg} / \mathrm{m}^{2}$ & $23.1(3.3)$ & $23.2(3.0)$ & $23.1(3.6)$ \\
\hline Current smoking, \% & 8.6 & 16.4 & 2.6 \\
\hline Current drinking, $\%$ & 40.3 & 61.8 & 23.9 \\
\hline Regular exercise, \% & 19.1 & 21.2 & 17.5 \\
\hline Antithrombotic use, $\%$ & 19.4 & 27.0 & 13.5 \\
\hline Antiplatelet use, $\%$ & 15.5 & 22.1 & 10.5 \\
\hline Anticoagulant use, \% & 5.3 & 8.1 & 3.2 \\
\hline APOE \& 2 carrier, $\%$ & 9.6 & 10.1 & 8.9 \\
\hline APOE \& 4 carrier, $\%$ & 18.9 & 21.2 & 17.2 \\
\hline History of symptomatic stroke, \% & 5.3 & 7.6 & 3.6 \\
\hline Ischemic stroke, \% & 3.9 & 5.6 & 2.6 \\
\hline Intracerebral hemorrhage, \% & 1.1 & 1.8 & 0.6 \\
\hline Subarachnoid hemorrhage, $\%$ & 0.3 & 0.2 & 0.4 \\
\hline Dementia, \% & 10.6 & 9.7 & 11.2 \\
\hline Lacunar infarction on MRI, \% & 22.2 & 30.1 & 16.1 \\
\hline $\begin{array}{l}\text { WMH (Fazekas grade } 2 \text { or } 3 \text { ) on MRI, } \\
\%\end{array}$ & 42.5 & 43.4 & 41.9 \\
\hline
\end{tabular}

Abbreviations: $\mathrm{BMI}=$ body mass index; $\mathrm{WMH}$ = white matter hyperintensity. Values are shown as means (SD) or frequencies.
The present study revealed that the prevalence of CMBs was $18.7 \%$ in elderly Japanese individuals aged $\geq 65$

years.

Table e-2 (links.lww.com/CPJ/A24) shows the prevalence of CMBs in $A P O E \& 4$ carriers and noncarriers. APOE $\varepsilon 4$ carriers were more likely to have lobar CMBs than noncarriers.

Since hypertension was a risk factor for CMBs in any location, the associations of $\mathrm{BP}$ levels and antihypertensive treatment status with the prevalence of $\mathrm{CMBs}$ are presented in table e-3 (links.lww.com/CPJ/A24). Users of antihypertensive agents were more likely to have deep/infratentorial and lobar CMBs, and elevated BP levels were associated with the presence of deep/infratentorial CMBs.

Finally, table e-4 (links.lww.com/CPJ/A24) shows the association of CMBs with other neurologic disorders such as symptomatic stroke (ischemic stroke and intracerebral hemorrhage), dementia, and ischemic small vessel diseases (lacunar infarction and WHM) on MRI. The presence of any, deep/infratentorial, and lobar CMBs were all significantly associated with these neurologic disorders.

\section{Discussion}

The present cross-sectional survey demonstrated that approximately 1 of 5 elderly people aged $\geq 65$ years had CMBs on a brain MRI in a general Japanese population. The prevalence of deep/infratentorial CMBs was higher than that of lobar CMBs. Older age and hypertension were associated with both deep/infratentorial and lobar CMBs. While lower serum cholesterol levels were a risk factor for deep/infratentorial CMBs, the $A P O E \& 4$ genotype and male sex were linked to the presence of lobar CMBs. The presence of CMBs was associated with other neurologic disorders such as symptomatic stroke, dementia, and ischemic small vessel diseases on MRI.

The present study revealed that the prevalence of CMBs was $18.7 \%$ in elderly Japanese individuals aged $\geq 65$ years. Several population-based studies have estimated the prevalence of CMBs in elderly populations, with the resulting prevalence of CMBs in the elderly ranging from $11.1 \%$ to $34.4 \%$ (table e-5, links.lww.com/CPJ/A24). ${ }^{9-13}$ These values were similar to the prevalence observed in the present study. The Sefuri Brain MRI Study, a population-based study for individuals aged $\geq 39$ years who lived in a rural village in Japan, reported that the prevalence of CMBs in Japanese elderly residents aged $>60$ years was $6.2 \%,{ }^{7}$ which was lower than ours, probably because the former study excluded individuals with a history of neurologic or 
Table 2 Age-specific prevalence of any, deep/infratentorial, and lobar cerebral microbleeds (CMBs), the Hisayama Study, 2012

\begin{tabular}{|c|c|c|c|c|c|c|c|c|c|c|}
\hline & \multirow{2}{*}{$\begin{array}{l}\text { No. of } \\
\text { participants }\end{array}$} & \multicolumn{3}{|c|}{ Any CMBs } & \multicolumn{3}{|c|}{ Deep/infratentorial CMBs } & \multicolumn{3}{|c|}{ Lobar CMBs } \\
\hline & & No. & $\%$ & $95 \% \mathrm{Cl}$ & No. & $\%$ & $95 \% \mathrm{Cl}$ & No. & $\%$ & $95 \% \mathrm{Cl}$ \\
\hline \multicolumn{11}{|l|}{ Total } \\
\hline Overall & 1,281 & 240 & 18.7 & $16.6-20.8$ & 173 & 13.5 & $11.6-15.4$ & 123 & 9.6 & $8.0-11.2$ \\
\hline $65-69 y$ & 356 & 42 & 11.8 & $8.4-15.2$ & 26 & 7.3 & $5.0-10.0$ & 25 & 7.0 & $4.3-9.7$ \\
\hline $70-74 y$ & 335 & 58 & 17.3 & $13.2-21.4$ & 40 & 11.9 & 8.4-15.4 & 29 & 8.7 & $5.7-11.7$ \\
\hline $75-79 y$ & 282 & 60 & 21.3 & $16.5-26.1$ & 46 & 16.3 & $12.0-20.6$ & 30 & 10.6 & $7.0-14.1$ \\
\hline $80-84 y$ & 188 & 45 & 23.9 & $17.8-30.0$ & 36 & 19.2 & $13.6-24.8$ & 20 & 10.6 & $6.2-15.0$ \\
\hline$\geq 85 y$ & 120 & 35 & 29.2 & $21.1-37.3$ & 25 & 20.8 & $13.5-28.1$ & 19 & 15.8 & $9.3-22.3$ \\
\hline$p$ for trend & & & & $<0.001$ & & & $<0.001$ & & & 0.001 \\
\hline \multicolumn{11}{|l|}{ Men } \\
\hline Overall & 556 & 128 & 23.0 & $19.5-26.5$ & 90 & 17.4 & $14.2-20.6$ & 71 & 12.8 & $10.0-15.6$ \\
\hline $65-69 y$ & 153 & 30 & 19.6 & $13.3-25.9$ & 20 & 13.1 & $7.8-18.4$ & 15 & 9.8 & $5.1-14.5$ \\
\hline $70-74 y$ & 148 & 25 & 16.9 & $10.9-22.9$ & 15 & 10.1 & $5.2-15.0$ & 17 & 11.5 & $6.4-16.6$ \\
\hline $75-79 y$ & 128 & 32 & 25.0 & $17.5-32.5$ & 27 & 21.1 & $14.0-28.2$ & 15 & 11.7 & $6.1-17.3$ \\
\hline $80-84 y$ & 78 & 23 & 29.5 & 19.4-39.6 & 17 & 21.8 & $12.6-31.0$ & 11 & 14.1 & $6.4-21.8$ \\
\hline$\geq 85 y$ & 49 & 18 & 36.7 & $23.2-50.2$ & 11 & 22.5 & $10.8-34.2$ & 13 & 26.5 & $14.1-38.9$ \\
\hline$p$ for trend & & & & 0.003 & & & 0.01 & & & 0.01 \\
\hline \multicolumn{11}{|l|}{ Women } \\
\hline Overall & 725 & 112 & 15.5 & $12.9-18.1$ & 83 & 11.9 & $9.5-14.3$ & 52 & 7.2 & $5.3-9.1$ \\
\hline $65-69 y$ & 203 & 12 & 5.9 & $2.7-9.1$ & 6 & 3.0 & $0.7-5.3$ & 10 & 4.9 & $1.9-7.9$ \\
\hline $70-74$ y & 187 & 33 & 17.7 & $12.2-23.2$ & 25 & 13.4 & 8.5-18.3 & 12 & 6.4 & $2.9-9.9$ \\
\hline $75-79 y$ & 154 & 28 & 18.2 & $12.1-24.3$ & 19 & 12.3 & $7.1-17.5$ & 15 & 9.7 & $5.0-14.4$ \\
\hline $80-84 y$ & 110 & 22 & 20.0 & $12.5-27.5$ & 19 & 17.3 & $10.2-24.4$ & 9 & 8.2 & $3.1-13.3$ \\
\hline$\geq 85 y$ & 71 & 17 & 23.9 & $14.0-33.8$ & 14 & 19.7 & $10.4-29.0$ & 6 & 8.5 & $2.0-15.0$ \\
\hline$p$ for trend & & & & $<0.001$ & & & $<0.001$ & & & 0.07 \\
\hline
\end{tabular}

Abbreviation: $\mathrm{Cl}=$ confidence interval.

psychiatric diseases, who were more likely to have CMBs than those without such a history. Some hospital- or institutionbased studies in Japan ${ }^{8,14,15,17}$ and Korea ${ }^{16}$ have also reported lower prevalence of CMBs $(3.1 \%-6.8 \%)$ than the present study because these studies recruited healthy volunteers and included younger participants.

The previous pathologic studies have shown that hypertensive angiopathy is mainly observed in deep or infratentorial brain areas, and amyloid angiopathy is mainly located in cerebral lobar regions. ${ }^{2}$ Some population-based observational studies have reported that hypertension ${ }^{5,8}$ or elevated $\mathrm{BP}^{8,9}$ was associated with deep/infratentorial CMBs. These findings were in accord with ours.
Intriguingly, the present study found a significant association between hypertension and lobar CMBs. A pathologic study failed to reveal a direct neuropathologic association between amyloid angiopathy and lobar CMBs. ${ }^{25}$ Another pathologic study also found that amyloid angiopathy and fibrinoid necrosis, which is one of the major findings of hypertensive angiopathy, coexisted in lobar CMBs. ${ }^{26}$ These findings raise the possibility that not only amyloid angiopathy, but also hypertensive angiopathy, contributes to the development of lobar CMBs. Population-based observational studies in Western populations also showed that hypertensive individuals tended to have a higher risk of the presence of lobar CMBs than normotensive individuals, but the associations did not quite reach a statistically significant 
Table 3 Association of risk factors with the presence of any cerebral microbleeds

\begin{tabular}{|c|c|c|c|c|c|c|}
\hline \multirow[b]{2}{*}{ Variables } & \multicolumn{3}{|c|}{ Age- and sex-adjusted } & \multicolumn{3}{|c|}{ Multivariable-adjusted } \\
\hline & OR & $95 \% \mathrm{Cl}$ & $p$ & OR & $95 \% \mathrm{Cl}$ & $p$ \\
\hline Age (per 10-y increase) & 1.65 & $1.35-2.03$ & $<0.001$ & 1.47 & $1.16-1.87$ & 0.002 \\
\hline Men & 1.70 & $1.28-2.26$ & $<0.001$ & 1.39 & $0.97-2.00$ & 0.08 \\
\hline Hypertension & 2.47 & $1.68-3.62$ & $<0.001$ & 2.19 & $1.44-3.33$ & $<0.001$ \\
\hline Diabetes mellitus & 1.29 & $0.93-1.79$ & 0.13 & 1.08 & $0.75-1.54$ & 0.69 \\
\hline $\begin{array}{l}\text { Serum total cholesterol (per } 1 \mathrm{mmol} / \mathrm{L} \\
\text { increase) }\end{array}$ & 0.89 & $0.75-1.05$ & 0.15 & 0.89 & $0.74-1.07$ & 0.22 \\
\hline BMI (per $1 \mathrm{~kg} / \mathrm{m}^{2}$ increase) & 1.03 & $0.99-1.08$ & 0.13 & 0.99 & $0.95-1.04$ & 0.80 \\
\hline Current smoking & 0.81 & $0.48-1.39$ & 0.45 & 1.13 & $0.63-2.03$ & 0.67 \\
\hline Current drinking & 1.18 & $0.86-1.63$ & 0.31 & 1.10 & $0.77-1.56$ & 0.60 \\
\hline Regular exercise & 0.86 & $0.59-1.25$ & 0.42 & 0.84 & $0.56-1.27$ & 0.41 \\
\hline Antithrombotic treatment & 1.57 & $1.12-2.21$ & 0.01 & 1.20 & $0.82-1.75$ & 0.35 \\
\hline APOE \& 2 carrier & 0.89 & $0.53-1.51$ & 0.67 & 0.94 & $0.55-1.61$ & 0.82 \\
\hline APOE $\varepsilon 4$ carrier & 1.46 & $1.02-2.10$ & 0.04 & 1.42 & $0.98-2.06$ & 0.06 \\
\hline
\end{tabular}

Abbreviations: $\mathrm{BMI}=$ body mass index; $\mathrm{Cl}=$ confidence interval; $\mathrm{OR}=$ odds ratio.

level. ${ }^{5,9}$ The exact reason for this discrepancy in the influence of hypertension on lobar vessels among populations is unknown, but it may be attributable to differences in the genetic backgrounds, age distributions, ${ }^{5}$ and lifestyles (e.g., salt intake) among populations. Our study failed to show a clear association between BP levels and lobar CMBs, probably because more than half of the participants were treated with antihypertensive agents.

In the present study, lower total cholesterol levels were associated with the presence of deep/infratentorial CMBs. Another study of Japanese individuals aged 27-95 years who

Table 4 Association of risk factors with the presence of deep/infratentorial cerebral microbleeds

\begin{tabular}{|c|c|c|c|c|c|c|}
\hline \multirow[b]{2}{*}{ Variables } & \multicolumn{3}{|c|}{ Age and sex-adjusted } & \multicolumn{3}{|c|}{ Multivariable-adjusted } \\
\hline & OR & $95 \% \mathrm{Cl}$ & $p$ & OR & $95 \% \mathrm{Cl}$ & $p$ \\
\hline Age (per 10-y increase) & 1.75 & $1.39-2.22$ & $<0.001$ & 1.49 & $1.14-1.95$ & 0.004 \\
\hline Men & 1.63 & $1.18-2.26$ & 0.003 & 1.20 & $0.79-1.82$ & 0.39 \\
\hline Hypertension & 2.67 & $1.69-4.21$ & $<0.001$ & 2.20 & $1.35-3.60$ & 0.002 \\
\hline Diabetes mellitus & 1.56 & $1.09-2.24$ & 0.02 & 1.37 & $0.92-2.02$ & 0.12 \\
\hline $\begin{array}{l}\text { Serum total cholesterol (per } 1 \mathrm{mmol} / \mathrm{L} \\
\text { increase) }\end{array}$ & 0.76 & $0.63-0.93$ & 0.01 & 0.78 & $0.63-0.97$ & 0.03 \\
\hline BMI (per 1 kg/m² increase) & 1.04 & $0.99-1.09$ & 0.15 & 0.99 & $0.94-1.05$ & 0.75 \\
\hline Current smoking & 0.93 & $0.51-1.68$ & 0.80 & 1.25 & $0.65-2.38$ & 0.51 \\
\hline Current drinking & 1.18 & $0.82-1.70$ & 0.38 & 1.13 & $0.75-1.68$ & 0.56 \\
\hline Regular exercise & 0.83 & $0.53-1.29$ & 0.40 & 0.84 & $0.53-1.34$ & 0.46 \\
\hline Antithrombotic treatment & 1.65 & $1.13-2.41$ & 0.01 & 1.19 & $0.78-1.82$ & 0.41 \\
\hline$A P O E$ \& 2 carrier & 0.83 & $0.45-1.53$ & 0.54 & 0.79 & $0.42-1.49$ & 0.47 \\
\hline APOE \&4 carrier & 1.04 & $0.67-1.62$ & 0.88 & 0.99 & $0.63-1.57$ & 0.97 \\
\hline
\end{tabular}

Abbreviations: $\mathrm{BMI}=$ body mass index; $\mathrm{Cl}$ = confidence interval; $\mathrm{OR}=$ odds ratio. 
In our participants, $A P O E \varepsilon 4$ was

a significant risk factor for lobar CMBs but not for deep/infratentorial CMBs.

underwent brain checkups with MRI also demonstrated a significant inverse association between serum total cholesterol levels and the likelihood of deep/infratentorial CMBs. ${ }^{8}$ Lower serum total cholesterol levels might induce the formation of microaneurysms in the brain through fragility of the vessel walls, resulting in the occurrence of CMBs. ${ }^{27}$ In support of this idea, a systematic review has shown that lower serum total cholesterol levels are significantly associated with hemorrhagic stroke. ${ }^{28}$ In contrast, epidemiologic studies conducted in Western populations found that lower cholesterol levels were associated with lobar CMBs, but not with deep/infratentorial CMBs. ${ }^{5,9}$ The reason for the discrepancy between Japanese and Western studies is unclear but may be attributable to differences in the genetic backgrounds, the age distributions, ${ }^{5}$ or the lower average levels of serum total cholesterol in Japanese compared to Western populations. ${ }^{9}$

In our participants, $A P O E \varepsilon 4$ was a significant risk factor for lobar CMBs but not for deep/infratentorial CMBs. The significant association of $A P O E \varepsilon 4$ with lobar CMBs was also observed in the Framingham Heart Study ${ }^{5}$ and the Rotterdam Study. ${ }^{9}$ A meta-analysis $^{29}$ reported that $A P O E \& 4$ was also significantly associated with cerebral amyloid angiopathy, which is considered to be one of the major causes of lobar CMBs. APOE $\varepsilon 4$ may induce cerebral amyloid angiopathy by increasing $\beta$-amyloid aggregation, impairing $\beta$-amyloid clearance, or both. ${ }^{30}$ On the other hand, the APOE $\varepsilon 2$ genotype was not associated with CMBs in the present study or in previous reports. ${ }^{9,10}$

Community-based studies have reported inconsistent results in regard to the sex difference in CMB prevalence..$^{5-7,9-12}$ Some studies have reported that men had higher prevalence of $\mathrm{CMBs}$ than women ${ }^{5,710,12}$ and others showed no sex difference. ${ }^{6,9,11}$ In the present study, the age-adjusted model indicated that men had a higher risk of both deep/infratentorial and lobar CMBs than women. However, the sex difference in deep/infratentorial CMBs was diminished after adjustment for multiple risk factors, probably because men were likely to have more cardiovascular risk factors than women. In contrast, the sex difference in lobar CMBs was unchanged and remained significant after multivariable adjustment, indicating that residual confounding factors may exist in the association of male sex with a risk of lobar CMBs.

We showed that deep/infratentorial CMBs were more frequent than lobar CMBs. A study based on healthy volunteers in Japan reported a similar finding. ${ }^{8}$ In contrast, some Western studies reported higher prevalence of lobar CMBs than deep/infratentorial CMBs. ${ }^{5,9-11}$ The reason for the discrepancy may be partially explained by the finding that Western people have a higher frequency of the APOE $\varepsilon 4$ genotype. $^{5,9,10}$

Table 5 Association of risk factors with the presence of lobar cerebral microbleeds

\begin{tabular}{|c|c|c|c|c|c|c|}
\hline \multirow[b]{2}{*}{ Variables } & \multicolumn{3}{|c|}{ Age-sex-adjusted } & \multicolumn{3}{|c|}{ Multivariable-adjusted } \\
\hline & OR & $95 \% \mathrm{Cl}$ & $p$ & OR & $95 \% \mathrm{Cl}$ & $p$ \\
\hline Age (per 10-y increase) & 1.58 & $1.21-2.07$ & $<0.001$ & 1.40 & $1.01-1.93$ & 0.04 \\
\hline Men & 2.00 & $1.37-2.93$ & $<0.001$ & 1.82 & $1.12-2.97$ & 0.02 \\
\hline Hypertension & 2.11 & $1.28-3.47$ & 0.003 & 1.89 & $1.09-3.28$ & 0.02 \\
\hline Diabetes mellitus & 0.94 & $0.59-1.48$ & 0.78 & 0.69 & $0.41-1.17$ & 0.17 \\
\hline $\begin{array}{l}\text { Serum total cholesterol (per } 1 \mathrm{mmol} / \mathrm{L} \\
\text { increase) }\end{array}$ & 0.92 & $0.73-1.15$ & 0.44 & 0.93 & $0.72-1.19$ & 0.54 \\
\hline BMI (per $1 \mathrm{~kg} / \mathrm{m}^{2}$ increase) & 1.03 & $0.97-1.09$ & 0.36 & 1.00 & $0.94-1.07$ & 0.99 \\
\hline Current smoking & 0.70 & $0.34-1.45$ & 0.34 & 1.03 & $0.48-2.23$ & 0.93 \\
\hline Current drinking & 1.11 & $0.72-1.69$ & 0.64 & 0.99 & $0.62-1.60$ & 0.99 \\
\hline Regular exercise & 0.89 & $0.54-1.46$ & 0.61 & 0.87 & $0.51-1.51$ & 0.63 \\
\hline Antithrombotic treatment & 1.87 & $1.22-2.88$ & 0.004 & 1.48 & $0.90-2.41$ & 0.12 \\
\hline APOE $\varepsilon 2$ carrier & 1.05 & $0.54-2.06$ & 0.88 & 1.16 & $0.59-2.31$ & 0.66 \\
\hline APOE \&4 carrier & 1.73 & $1.09-2.74$ & 0.02 & 1.70 & $1.08-2.80$ & 0.02 \\
\hline
\end{tabular}

Abbreviations: $\mathrm{BMI}=$ body mass index; $\mathrm{Cl}$ = confidence interval; $\mathrm{OR}=$ odds ratio. 
The present study is the largest observational study to explore the prevalence of and risk factors for CMBs in a Japanese general elderly population. However, some limitations should be considered. First, although the participation rate of the present study was relatively high, approximately onethird of the residents did not undergo MRI examination. The individuals excluded from the study were likely to be older and to have more unhealthy backgrounds than those included in the study (table e-6, links.lww.com/CPJ/A24). Therefore, the prevalence of CMBs and the association between each risk factor and CMBs reported in this study might be underestimated. Second, because the present study was conducted in a cross-sectional manner, we cannot provide definite information about causal associations. Finally, we could not classify the cerebellar CMBs, which seemed to have different characteristics from other CMBs, because of limited sample size.

The present study suggests that approximately 1 of 5 elderly Japanese has some type of $\mathrm{CMB}$ and that individuals with CMBs have higher risk of symptomatic stroke and dementia. Deep/infratentorial CMBs and lobar CMBs have different risk factors, reflecting different pathologic backgrounds. Further prospective studies are needed to elucidate risk factors for the development of CMBs and to establish strategies for prevention of $\mathrm{CMBs}$ and subsequent neurologic disorders, including symptomatic stroke and dementia.

\section{Author contributions}

Tomohiro Yubi: study concept and design, analysis and interpretation of data, acquisition of data, writing of the manuscript. Jun Hata: study concept and design, interpretation of data, acquisition of data, critical revision of the manuscript for intellectual content. Tomoyuki Ohara: interpretation of data, acquisition of data, critical revision of the manuscript for intellectual content. Naoko Mukai, interpretation of data, acquisition of data, critical revision of the manuscript for intellectual content. Yoichiro Hirakawa: interpretation of data, acquisition of data, critical revision of the manuscript for intellectual content. Daigo Yoshida: interpretation of data, acquisition of data, critical revision of the manuscript for intellectual content. Seiji Gotoh: interpretation of data, acquisition of data, critical revision of the manuscript for intellectual content. Naoki Hirabayashi: interpretation of data, acquisition of data, critical revision of the manuscript for intellectual content. Yoshihiko Furuta: interpretation of data, acquisition of data, critical revision of the manuscript for intellectual content. Tetsuro Ago: interpretation of data, critical revision of the manuscript for intellectual content. Takanari Kitazono: interpretation of data, critical revision of the manuscript for intellectual content, study supervision. Yutaka Kiyohara: study concept and design, interpretation of data, acquisition of data, critical revision of the manuscript for intellectual content, study supervision. Toshiharu Ninomiya: study concept and design, interpretation of data, acquisition of data, critical revision of the manuscript for intellectual content, study supervision.

\section{Acknowledgment}

The authors thank the staff of the Division of Health and Welfare of Hisayama for cooperation in this study; and Professor Yoshinao Oda, Professor Toru Iwaki, and the staff of the Department of Anatomic Pathology and Department of Neuropathology, Graduate School of Medical Sciences, Kyushu University, who provided insight and expertise in regard to the autopsy findings that assisted this research.

\section{Study funding}

Study funded by Grants-in-Aid for Scientific Research (A) (JP16H02644, and JP16H02692), (B) (JP16H05850, JP16H05557, and JP17H04126), and (C) (JP15K09267, JP15K08738, JP15K09835, JP16K09244, JP17K09114, JP17K09113, and JP17K01853) from the Ministry of Education, Culture, Sports, Science and Technology of Japan; by Health and Labour Sciences Research Grants of the Ministry of Health, Labour and Welfare of Japan (H25-Junkankitou [Seishuu]-Sitei-022, H29-Junkankitou [Seishuu]-Ippan-003, and H27-Shokuhin-[Sitei]-017); and by the Japan Agency for Medical Research and Development (dk0207025, ek0210082, gm0610007, ek0210083, km0405202, and ek0210080).

\section{Disclosure}

T. Yubi, J. Hata, T. Ohara, N. Mukai, Y. Hirakawa, D. Yoshida, S. Gotoh, N. Hirabayashi, Y. Furuta, and T. Ago report no disclosures. T. Kitazono has received funding for travel or speaker honoraria from Daiichi Sankyo Co., Ltd., Bayer, and Yakuhin, Ltd.; and receives research support from Daiichi Sankyo Co., Ltd., Takeda Pharmaceutical Co., Ltd., Astellas Pharma Inc., Chugai Pharmaceutical Co., Ltd., MSD, K.K. Nippon, and Boehringer Ingelheim Co., Ltd. Y. Kiyohara and T. Ninomiya report no disclosures. Full disclosure form information provided by the authors is available with the full text of this article at Neurology.org/cp.

Received November 29, 2017. Accepted in final form March 28, 2018.

\section{References}

1. Offenbacher H, Fazekas F, Schmidt R, Koch M, Fazekas G, Kapeller P. MR of cerebral abnormalities concomitant with primary intracerebral hematomas. Am J Neuroradiol 1996;17:573-578.

2. Cordonnier C, Al-Shahi Salman R, Wardlaw J. Spontaneous brain microbleeds: systematic review, subgroup analyses and standards for study design and reporting. Brain 2007;130:1988-2003.

3. Ioannidis JP, Ntzani EE, Trikalinos TA. "Racial" differences in genetic effects for complex diseases. Nat Genet 2004;36:1312-1318.

4. Hata J, Kiyohara Y. Epidemiology of stroke and coronary artery disease in Asia. Circ J 2013;77:1923-1932.

5. Romero JR, Preis SR, Beiser A, et al. Risk factors, stroke prevention treatments, and prevalence of cerebral microbleeds in the Framingham Heart Study. Stroke 2014;45: $1492-1494$.

6. Roob G, Schmidt R, Kapeller P, Lechner A, Hartung HP, Fazekas F. MRI evidence of past cerebral microbleeds in a healthy elderly population. Neurology 1999;52:991-994.

7. Takashima $\mathrm{Y}$, Mori $\mathrm{T}$, Hashimoto $\mathrm{M}$, et al. Clinical correlating factors and cognitive function in community-dwelling healthy subjects with cerebral microbleeds. J Stroke Cerebrovasc Dis 2011;20:105-110.

8. Mitaki S, Nagi A, Oguro H, Yamaguchi S. Serum lipid fractions and cerebral micro bleeds in a healthy Japanese population. Cerebrovasc Dis 2017;43:186-191.

9. Vernooij MW, van der Lugt A, Ikram MA, et al. Prevalence and risk factors of cerebral microbleeds: the Rotterdam Scan Study. Neurology 2008;70:1208-1214.

10. Sveinbjornsdottir S, Sigurdsson S, Aspelund T, et al. Cerebral microbleeds in the population based AGES-Reykjavik Study: prevalence and location. J Neurol Neurosurg Psychiatry 2008;79:1002-1006.

11. Wiegman AF, Meier IB, Schupf N, et al. Cerebral microbleeds in a multiethnic elderly community: demographic and clinical correlates. J Neurol Sci 2014;345:125-130. 
12. Gustavsson AM, Stomrud E, Abul-Kasim K, et al. Cerebral microbleeds and white matter hyperintensities in cognitively healthy elderly: a cross-sectional cohort study evaluating the effect of arterial stiffness. Cerebrovasc Dis Extra 2015;5:41-51.

13. Hilal S, Mok V, Youn YC, Wong A, Ikram MK, Chen CL. Prevalence, risk factors and consequences of cerebral small vessel diseases: data from three Asian countries. J Neurol Neurosurg Psychiatry 2017;88:669-674.

14. Igase $M$, Tabara $Y$, Igase $K$, et al. Asymptomatic cerebral microbleeds seen in healthy subjects have a strong association with asymptomatic lacunar infarction. Circ J 2009;73:530-533.

15. Tsushima Y, Tanizaki Y, Aoki J, Endo K. MR detection of microhemorrhages in neurologically healthy adults. Neuroradiology 2002;44:31-36.

16. Jang SH, Lee H, Kim JS, et al. Association between helicobacter pylori infection and cerebral small vessel disease. Korean J Fam Med 2015;36:227-232.

17. Yakushiji Y, Nishiyama M, Yakushiji S, et al. Brain microbleeds and global cognitive function in adults without neurological disorder. Stroke 2008;39:3323-3328.

18. Hata J, Ninomiya T, Hirakawa Y, et al. Secular trends in cardiovascular disease and its risk factors in Japanese half-century data from the Hisayama Study (1961-2009). Circulation 2013;128:1198-1205.

19. Hirabayashi N, Hata J, Ohara T, et al. Association between diabetes and hippocampal atrophy in elderly Japanese: the Hisayama Study. Diabetes Care 2016;39: $1543-1549$.

20. Fazekas F, Chawluk JB, Alavi A, Hurtig HI, Zimmerman RA. MR signal abnormalities at 1.5 T in Alzheimer's dementia and normal aging. Am J Roentgenol 1987; 149:351-356.
21. Ohnishi Y, Tanaka T, Ozaki K, Yamada R, Suzuki H, Nakamura Y. A high-throughput SNP typing system for genome-wide association studies. J Hum Genet 2001;46:471-477.

22. Ohara T, Ninomiya T, Kubo M, et al. Apolipoprotein genotype for prediction of Alzheimer's disease in older Japanese: the Hisayama Study. J Am Geriatr Soc 2011;59:1074-1079.

23. American Psychiatric Association. Diagnostic and Statistical Manual of Mental Disorders, 3rd ed. Washington, DC: American Psychiatric Association; 1987.

24. Ohara T, Hata J, Yoshida D, et al. Trends in dementia prevalence, incidence, and survival rate in a Japanese community. Neurology 2017;16:1925-1932.

25. Kövari E, Charidimou A, Herrmann FR, Giannakopoulos P, Bouras C, Gold G. No neuropathological evidence for a direct topographical relation between microbleeds and cerebral amyloid angiopathy. Acta Neuropathol Commun 2015;3:49.

26. van Veluw SJ, Biessels GJ, Klijn CJ, Rozemuller AJ. Heterogeneous histopathology of cortical microbleeds in cerebral amyloid angiopathy. Neurology 2016;86:867-871.

27. Konishi M, Iso H, Komachi $\mathrm{Y}$, et al. Associations of serum total cholesterol, different types of stroke, and stenosis distribution of cerebral arteries the Akita Pathology Study. Stroke 1993;24:954-964

28. Wang X, Dong Y, Qi X, Haung C, Hou L. Cholesterol levels and risk of hemorrhagic stroke: a systematic review and meta-analysis. Stroke 2013;44:1833-1839.

29. Rannikmae K, Samarasekera N, Martinez-Gonzalez NA, Al-Shahi Salman R, Sudlow CL. Genetics of cerebral amyloid angiopathy: systematic review and meta-analysis. J Neurol Neurosurg Psychiatry 2013;84:901-908.

30. Verghese PB, Castellano JM, Holtzman DM. Apolipoprotein E in Alzheimer's disease and other neurological disorders. Lancet Neurol 2011;10:241-252.

\section{Share Your Insights, Expertise, and Experiences}

- How are you employing drugs and devices in your field?

- What ethical challenges do you face?

- Do you have a case report that is illustrative of a clinical challenge?

- What challenges have you faced or successes have you enjoyed in bringing greater efficiency to your practice?

Deliver a high-quality, peer-reviewed message to your colleagues in practice, submit your paper at NPub.org/NCP/submit. 